\title{
ÉTUde DE LA PROPAGATION DE FISSURES PAR FATIGUE DANS DES ACIERS INOXYDABLES AUSTÉNITIQUES A BAS CARBONE DU TYPE 304L ET 316L
}

\author{
B. YAHIAOUI $*$ et P. PETREQUIN ** \\ C.E.N. de Saclay. BP no 2, 91190 Gif-sur-Yvette, France
}

Résumé. - On a étudié à la température ambiante la propagation de fissures par fatigue dans les aciers austénitiques du type Z2CN1810 (304L) et Z2CND1712 (316L).

Les principaux résultats sont les suivants :

- Les vitesses de propagation des fissures par fatigue suivent globalement la loi de Paris

$$
\frac{\mathrm{d}^{2} a}{\mathrm{~d} N}=C(\Delta K)^{n}
$$

- Dans les mêmes conditions de sollicitations, l'acier 304L présente une plus faible résistance à la propagation de fissure par fatigue que l'acier $316 \mathrm{~L}$.

- L'influence de la contrainte moyenne est complexe. Il semble que la longueur de fissure intervienne d'une manière plus importante que ne le prédit la loi de Paris. Une formulation du type

$$
\frac{\mathrm{d}^{2} a}{\mathrm{~d} N}=C(R)(\Delta K)^{n} a^{P}
$$

semblerait mieux adaptée.

- Par comparaison aux résultats présentés par divers auteurs concernant les aciers à carbone normal (304) on note une vitesse de propagation plus élevée dans les aciers à bas carbone étudiés ici (304L). La variation du facteur d'intensité de contrainte $\left(\Delta K_{0}\right)$ conduisant à une vitesse de propagation de $10^{-5} \mathrm{~cm}_{\text {cycle }}^{-1}$ est voisine de $23 \mathrm{MP} a \sqrt{\mathrm{m}}$ pour le $304 \mathrm{~L}$ et $27-28$ pour le 304.

- On observe pour les 2 matériaux un seuil de non-propagation (après $5 \times 10^{6}$ cycles) par fatigue $\left(\Delta K_{\mathrm{s}}\right)$. Ce seuil est généralement bas $(5$ à $10 \mathrm{MP} a \sqrt{\mathrm{m}})$. Il dépend d'une manière linéairement décroissante de $R$ et est plus élevé pour l'acier 316L que pour l'acier 304L.

- La fissuration est généralement transcristalline. On rencontre cependant des zones de rupture intergranulaire qui correspondent à de très faibles vitesses de propagation c'est-à-dire proches des zones d'arrêt déterminant le $\Delta K_{\mathrm{s}}$.

Abstract. - The fatigue crack growth at room temperature in low carbon austenitic stainless steels 304L and 316L, has been studied. The main results are the following :

- Fatigue crack growth rate obey the Paris Law :

$$
\frac{\mathrm{d}^{2} a}{\mathrm{~d} N}=C(\Delta K)^{n} .
$$
steel.

- Under the same sollicitations, fatigue crack growth rate is higher for $304 \mathrm{~L}$ steel than for $316 \mathrm{~L}$

- The influence of the mean stress ( $R$ ratio) is quite complex. The effect of the crack length seems to be higher than predicted by the Paris Law.

Another formulation :

$$
\frac{\mathrm{d}^{2} a}{\mathrm{~d} N}=C(R)(\Delta K)^{n} a^{P}
$$

should be better.

- By the comparison to the literature, the low carbon austenitic steels (304L) studied here, present a higher crack growth than normal carbon austenitic steels (304).

The values of the stress intensity factor variation, leading to a crack growth rate of $10^{-5} \mathrm{c} / \mathrm{cycle}$ at $R=0$, are $23 \mathrm{MP} a \sqrt{\mathrm{m}}$ for 304L steel and 27-28 for 304 steels.

On both materials studied, a threshold of non-propagation after $2 \times 10^{6}$ cycles $\left(\Delta K_{\mathrm{s}}\right)$ has been observed. This threshold is quite low (5 to $10 \mathrm{MP} a \sqrt{\mathrm{m}}$ ). It depends linearly of the $R$ ratio and is higher for $316 \mathrm{~L}$ steel than for $304 \mathrm{~L}$ steel.

- The cracks are generally transcrystalline. However, intergranular cracking has been observed in some few cases at very low crack growth rates near the arrest zones in the $\Delta K_{\mathrm{s}}$ experiments.

(*) Stagiaire au Département de Technologie, Service de Recherches Métallurgiques Appliquées.

$\left(^{* *}\right)$ Ingénieur au Département de Technologie, Service de Recherches Métallurgiques Appliquées. 
1. Introduction. - La construction de réacteurs utilise des quantités importantes d'aciers inoxydables austénitiques sous forme de cuves de grandes dimensions et de tuyauteries.

Afin de juger de la sûreté de ces installations, il est essentiel de connaître les cinétiques de croissance de fissures ou défauts éventuels en relation avec les sollicitations des structures. On examine ici la croissance de fissures dans les aciers austénitiques par fatigue. On cherche en particulier à préciser les points suivants :

1.1 Quelle est la cinétique de croissance en fatigue d'un défaut existant ? étude de la vitesse de propagation en fonction des sollicitations.

1.2 Dans quelles conditions de sollicitations un défaut existant cesse-t-il de se propager ? étude d'un seuil de propagation.

1.3 Comment un défaut peut-il se créer par fatigue ? étude de l'initiation.

Ces points sont abordés pour deux aciers inoxydables austénitiques à bas carbone du type $\mathrm{Z} 2 \mathrm{CN} 1810$ (304L) et Z2CND 1712 (316L) sollicités à la température ambiante, pour lesquels aucune donnée n'existe actuellement dans la littérature et qui sont largement utilisés en France.

2. Méthodes utilisées. Conditions expérimentales. Pour l'analyse des cinétiques de propagation et des conditions d'initiation et d'arrêt des fissures on a utilisé les méthodes déduites de la mécanique de la rupture linéaire élastique. Ces études ont été complétées par des examens des surfaces de rupture.

2.1 MatériauX ÉTUdiés. - Les compositions chimiques des deux aciers $304 \mathrm{~L}$ et $316 \mathrm{~L}$ étudiés sont présentées dans le tableau I.

Les caractéristiques mécaniques à la température ambiante sont indiquées dans le tableau II.
Il s'agit de tôles bien hypertrempées d'épaisseur $25 \mathrm{~mm}$ et $70 \mathrm{~mm}$ pour les aciers $304 \mathrm{~L}$ et $316 \mathrm{~L}$ respectivement.

Les tailles de grain des matériaux sont de $55 \mu$ (5-6 Afnor) pour le 304L et de $100 \mu$ (3-4 Afnor) pour le $316 \mathrm{~L}$.

2.2 CONDITIONS EXPÉRIMENTALES. - 2.2.1 Eprouvettes. - On a utilisé des éprouvettes à trou central et à fissure traversante de dimension $500 \times 100 \times 25 \mathrm{~mm}^{3}$ ou $500 \times 100 \times 16 \mathrm{~mm}^{3}$ illustrées sur la figure 1 .

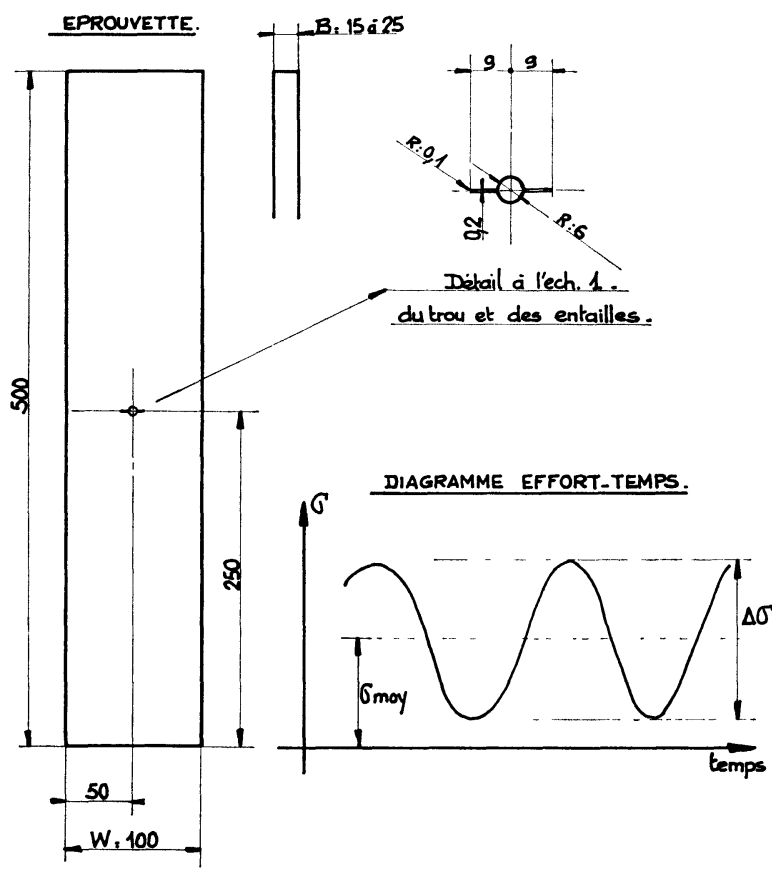

Fig. 1. $-\Delta K=\Delta \sigma\left(\frac{W}{1-v^{2}} \operatorname{tg} \frac{\pi a}{W}\right)^{1 / 2}$

$K$ : Facteur d'intensité de contrainte.

$W$ : Largeur de l'éprouvette.

$v$ : Coefficient de Poisson.

$2 a$ : Longueur de la fissure.

Eprouvette utilisée et formule de calibration.

\section{TABleAU I}

Composition chimique

$\begin{array}{ccccccccccccc}\text { Matériau } & \begin{array}{c}\mathrm{C} \\ \mathrm{ppm}\end{array} & \mathrm{Cr} & \mathrm{Ni} & \mathrm{Mo} & \mathrm{Mn} & \mathrm{Si} & \begin{array}{c}\mathrm{S} \\ \mathrm{ppm}\end{array} & \begin{array}{c}\mathrm{O}^{2} \\ \mathrm{ppm}\end{array} & \begin{array}{c}\mathrm{N}^{2} \\ \mathrm{ppm}\end{array} & \mathrm{P} & \mathrm{B} & \mathrm{Ti} \\ 304 \mathrm{~L} & \overline{160} & \overline{18,7} & 1 \overline{1} & \overline{0,34} & \overline{1,13} & \overline{0,46} & \overline{320} & \overline{177} & \overline{457} & 0, \overline{026} & - & - \\ 316 \mathrm{~L} & 206 & 16,3 & 13,1 & 2,17 & 1,55 & 0,45 & 147 & 164 & 248 & 0,019 & 0,001 & \leqslant 0,003\end{array}$

TABLEAU II

Caractéristiques mécaniques

\begin{tabular}{|c|c|c|c|c|c|}
\hline Matériau & & $R_{E} \mathrm{~kg} / \mathrm{mm}^{2}$ & $R_{\max } \mathrm{kg} / \mathrm{mm}^{2}$ & A $\%$ & $\%$ Striction \\
\hline \multirow{2}{*}{$304 \mathrm{~L}$} & Sens long & 23,5 & 62 & 66 & \\
\hline & Sens travers & 24,8 & 62 & 67 & \\
\hline \multirow{2}{*}{$316 \mathrm{~L}$} & Sens long & 25,5 & 54,7 & 64,2 & 51,5 \\
\hline & Sens travers & 25,9 & 55,2 & 64,5 & 51 \\
\hline
\end{tabular}


Le trou central a un diamètre de $6 \mathrm{~mm}$ prolongé par deux entailles de $6 \mathrm{~mm}$ de longueur et $0,2 \mathrm{~mm}$ d'épaisseur terminées par un rayon de $0,1 \mathrm{~mm}$, réalisées par électro-érosion.

La calibration de ces éprouvettes est obtenue par la formule d'Irwin-Westergaard, en déformation plane

$$
K=\left(\frac{W}{1-v^{2}}\right)^{1 / 2} \sigma\left(\operatorname{tg} \frac{\pi a}{W}\right)^{1 / 2}
$$

où $W$ représente la largeur de l'éprouvette, $2 a$, la longueur de la fissure, $\sigma$ la contrainte brute appliquée et $v$ le coefficient de Poisson du matériau.

On a comparé cette expression à d'autres formules de calibration :

$$
\begin{aligned}
K=\sigma a^{1 / 2}[1,77 & +0,227\left(\frac{2 a}{W}\right)- \\
& \left.\quad-0,510\left(\frac{2 a}{W}\right)^{2}+2,7\left(\frac{2 a}{W}\right)^{3}\right]
\end{aligned}
$$

et

$$
K=\sigma W^{1 / 2}\left(\frac{\pi a}{W} \sec \frac{\pi a}{W}\right)^{1 / 2}
$$

L'écart entre ces différentes expressions reste inférieur à $5 \%$ et c'est la formule d'Irwin qui a été utilisée dans toute cette étude.

Dans les cas où la quantité de métal n'était pas suffisante, des ailes ont été ajoutées par soudage aux coupons d'essai de $100 \times 100 \mathrm{~mm}^{2}$ afin de constituer l'éprouvette de $500 \times 100 \times 16 \mathrm{~mm}^{3}$.

2.2.2 Essais. - Les éprouvettes sont sollicitées en traction répétée sur une machine hydraulique asservie de $\pm 60 \mathrm{t}$ (Losenhausen). L'essai est effectué à charge contrôlée. La sollicitation cyclique est une sinusoïde superposée à une valeur moyenne fixe pendant tout l'essai.

Les fréquences utilisées sont comprises entre 16 et $20 \mathrm{~Hz}$. La longueur de fissure est mesurée optiquement sur la surface préalablement polie de l'éprouvette à l'aide d'un microscope monté sur un plateau mobile par vis micrométriques. Pour améliorer la mesure en cours d'essai, l'éclairage est réalisé par un stroboscope à faisceau étroit synchronisé avec la charge maximale, c'est-à-dire avec l'ouverture maximale de la fissure.
Au cours d'un essai aucun paramètre extérieur : charge moyenne, amplitude, n'est modifié sauf pour l'initiation de la fissure de fatigue.

La rupture finale se produit par instabilité plastique, quand la contrainte dans la section restante de l'éprouvette atteint la charge de rupture de l'acier.

On a examiné l'influence de la charge moyenne et de l'amplitude. Les conditions d'essais sont regroupées dans le tableau III.

On a utilisé pour classer les résultats la notation

$$
R=\frac{\sigma_{\min }}{\sigma_{\max }}=\frac{K_{\min }}{K_{\max }}
$$

les indices min et $\max$ correspondent aux valeurs minimales et maximales atteintes par la contrainte $\sigma$ ou le facteur d'intensité de contrainte $K$ au cours d'un cycle de fatigue. Les principales grandeurs utilisées sont liées par les relations :

$$
\begin{aligned}
\Delta K & =K_{\max }(1-R) \quad \Delta K: \text { amplitude de } K . \\
K_{\text {moy }} & =K_{\max }\left(\frac{1+R}{2}\right) \quad K_{\text {moy }}: \text { valeur moyenne de } K . \\
& =\frac{\Delta K}{2} \frac{1+R}{1-R}
\end{aligned}
$$

3. Cinétique de propagation. - 3.1 RÉsultats. 3.1.1 Acier 304L. - Les résultats sont présentés sur les figures 2 et 3 . Les mêmes résultats sont exprimés en fonction de $\Delta K$ sur les figures 4 et 5 .

On constate bien que globalement on obtient une loi du type de celle de Paris :

$$
\frac{\mathrm{d}^{2} a}{\mathrm{~d} N}=C(\Delta K)^{n}=10^{-5}\left(\frac{\Delta K}{\Delta K_{0}}\right)^{n}
$$

$\Delta K_{0}$ correspond à une vitesse de propagation de $10^{-5} \mathrm{~cm} \mathrm{cycle}^{-1}$.

Les unités utilisées sont le $\mathrm{cm}$ et le $\mathrm{MP} a \sqrt{\mathrm{m}}$ ou hbar $\sqrt{\mathrm{cm}}$. Pour les essais à $R$ faible on note nettement

\begin{tabular}{|c|c|c|c|c|c|c|}
\hline & $\sigma_{\mathrm{m}}$ & 7 & 7 & 7 & 5 & 9 \\
\hline $316 \mathrm{~L}$ & $\Delta \sigma$ & 7 & 9 & 11 & 10 & 7 \\
\hline & $R$ & 0,334 & 0,217 & 0,12 & 0 & \\
\hline
\end{tabular}
3 phases de propagation.

Les paramètres de la loi de Paris déterminés par la méthode des moindres carrés et corrigés pour obtenir un exposant constant sont regroupés sur le tableau IV.

L'exposant moyen obtenu est de 4,315.

\section{TABleaU III}

\section{Récapitulatif des essais effectués}

$\begin{array}{lllllrrrrrrrr} & \sigma_{\mathrm{m}} & 7 & 7 & 7 & 7 & 5 & 9 & 8 & 7 & 6 & 5 & 3,5 \\ 304 \mathrm{~L} & \Delta \sigma & 5 & 7 & 9 & 11 & 10 & 7 & 7 & 7 & 7 & 7 & 7 \\ & R & 0,475 & 0,334 & 0,217 & 0,12 & 0 & 0,44 & 0,39 & 0,334 & 0,264 & 0,176 & 0\end{array}$




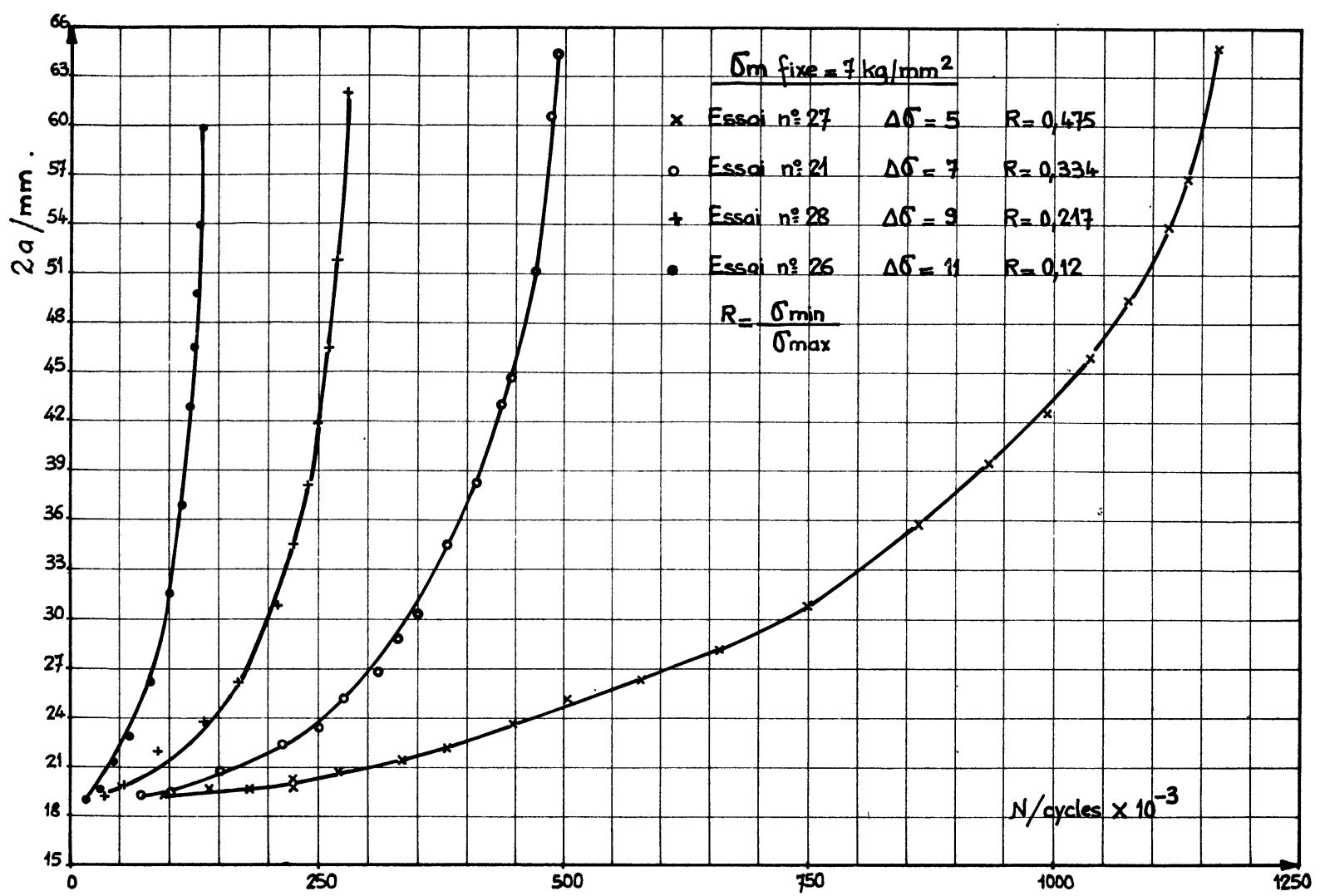

FIG. 2. - Propagation de fissures. Essai à contrainte moyenne fixe. Acier 304L.
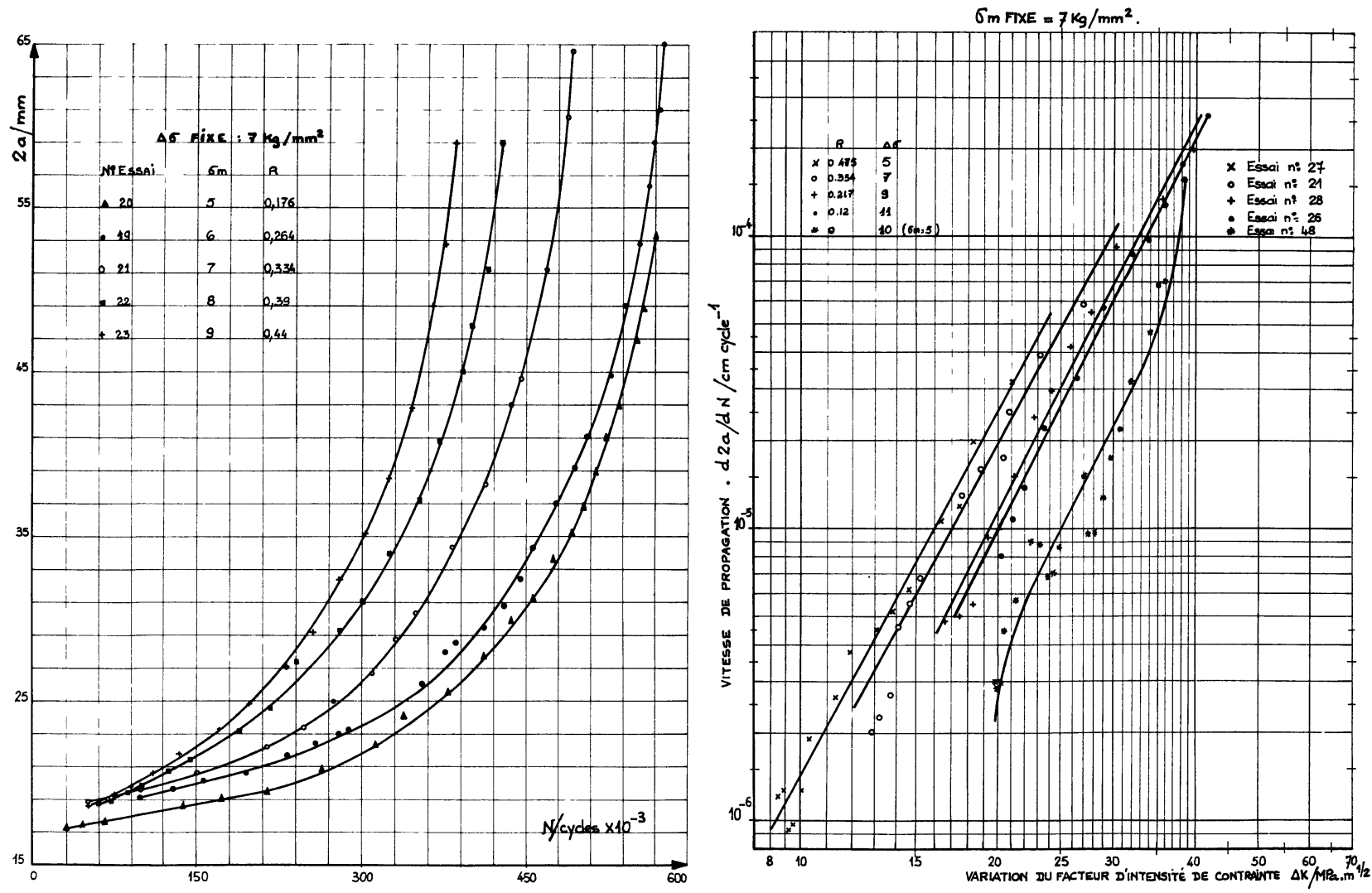

Fig. 3. - Propagation de fissures. Essais à amplitude fixe. Fig. 4. - Expression de la loi de Paris. Essais à contrainte Acier 304L. moyenne fixe. Acier 304L. 


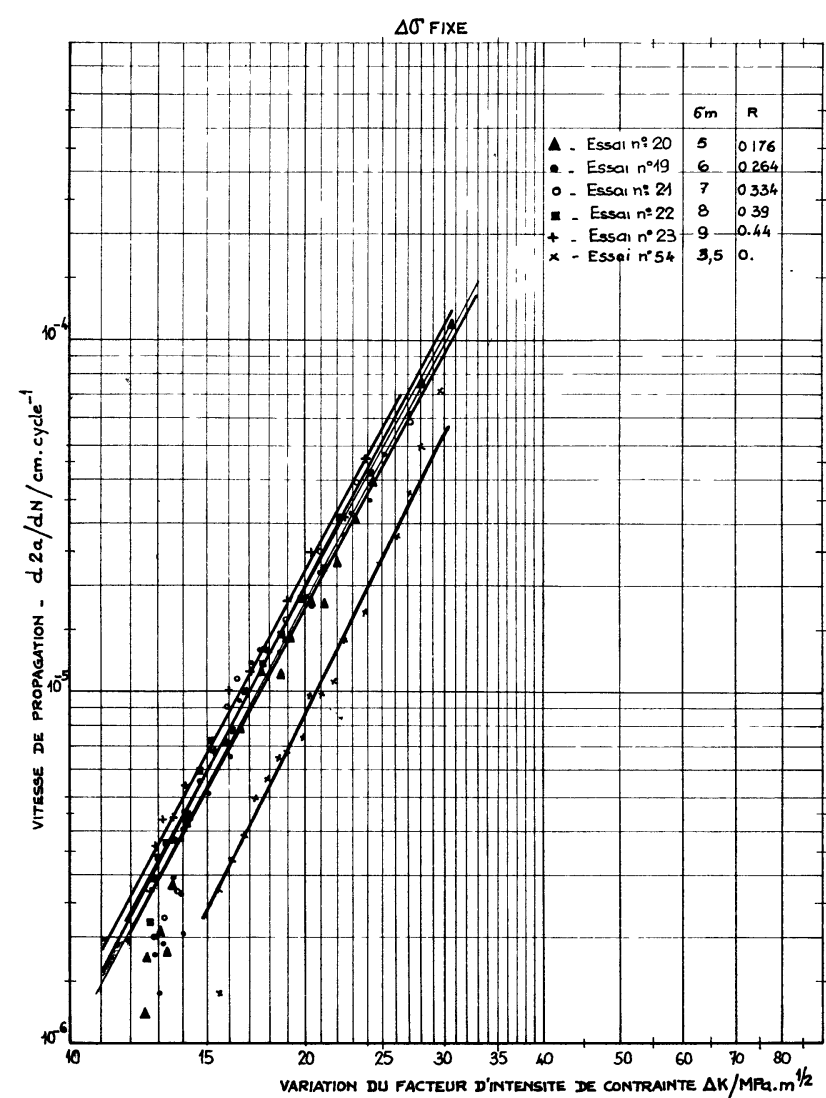

FIG. 5. - Expression de la loi de Paris. Essais à amplitude fixe. Acier 304L.

TABleau IV

Acier 304L : principaux résultats

$$
\mathrm{d}^{2} a / \mathrm{d} N=C \Delta K^{n} \quad n=4,315
$$

unités $\mathrm{d}^{2} a / \mathrm{d} N / \mathrm{cm}$ cycle $^{-1}$

\begin{tabular}{|c|c|c|c|}
\hline & $\mathrm{MPa}$ & & \\
\hline & $R$ & $C \times 10^{11}$ & $\Delta K_{0}$ \\
\hline & 0 & 1,987 & 20,95 \\
\hline & 0,176 & 4,20 & 17,68 \\
\hline$\Delta \sigma=7 \mathrm{~kg} / \mathrm{mm}^{2}$ & 0,264 & 4,33 & 17,49 \\
\hline & 0,334 & 4,79 & 17,09 \\
\hline & 0,39 & 5,03 & 16,90 \\
\hline & 0,44 & 5,63 & 16,46 \\
\hline & 0,12 & 2,41 & 20,04 \\
\hline & 0,217 & 2,78 & 19,38 \\
\hline $\mathrm{n}=7 \mathrm{~kg} / \mathrm{mm}^{2}$ & 0,334 & 4,79 & 17,09 \\
\hline & 0,475 & 6,36 & 16 \\
\hline $\mathrm{n}=5 \mathrm{~kg} / \mathrm{mm}^{2}$ & 0 & 0,91 & 25,11 \\
\hline
\end{tabular}

3.1.2 Acier $316 L$. - Les résultats sont présentés sur la figure 6 et dans le tableau V. L'exposant est sensiblement plus élevé : $\sim 5,7$.

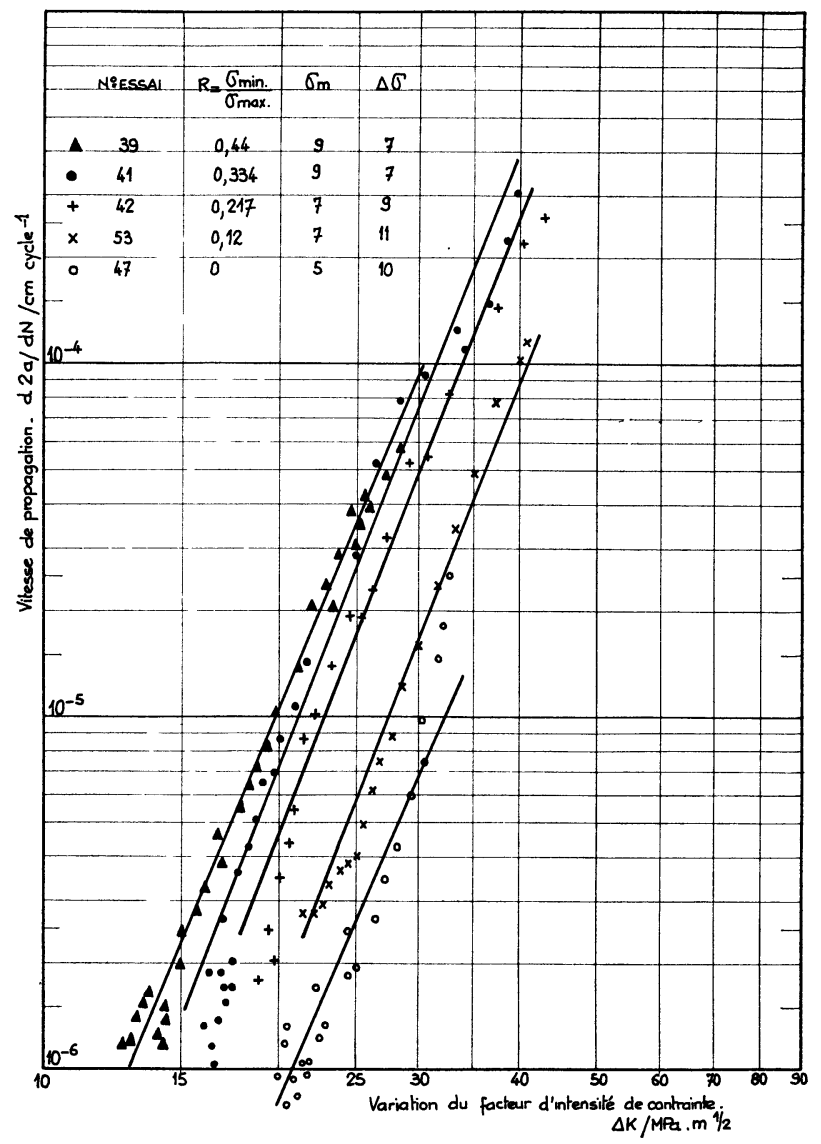

Fig. 6. - Expression de la loi de Paris. Acier 316L.

\section{TABLEAU V}

Acier 316L : principaux résultats

\begin{tabular}{lccl}
\multicolumn{1}{c}{$R$} & $C \times 10^{13}$ & $n$ & $\Delta K_{0}$ \\
0 & $\overline{-}$ & $\frac{n}{4}$ & 32 \\
0,12 & 0,4 & 5,71 & 28,5 \\
0,217 & 1,6 & 5,73 & 22,5 \\
0,334 & 2,19 & 5,77 & 21 \\
0,44 & 3,88 & 5,70 & 20,2
\end{tabular}

3.2 Discussion. - 3.2.1 Différence de comportement entre les aciers $304 \mathrm{~L}$ et $316 \mathrm{~L}$. - On note que les fissures se propagent sensiblement moins vite dans l'acier 316L que dans l'acier 304L. Pour les faibles $\Delta K(\sim 20 \mathrm{Mp} a \sqrt{\mathrm{m}})$ on note une variation voisine du simple au double. Aux forts $\Delta K$ les vitesses tendent à se rejoindre du fait de l'exposant plus élevé obtenu pour l'acier 316L.

Ce résultat semble différent de celui observé par Shahinian [3-1] qui ne note aucune différence de vitesse de propagation entre l'acier 316 et l'acier 304.

3.2.2 Comparaison à d'autres résultats. - Les seuls résultats dont on dispose dans la littérature concernent les aciers à carbone normal 304 et 316 . Ce sont principalement les résultats de Brothers [5], de Shahinan [3-4], de James [6], de Henry [7] et de 


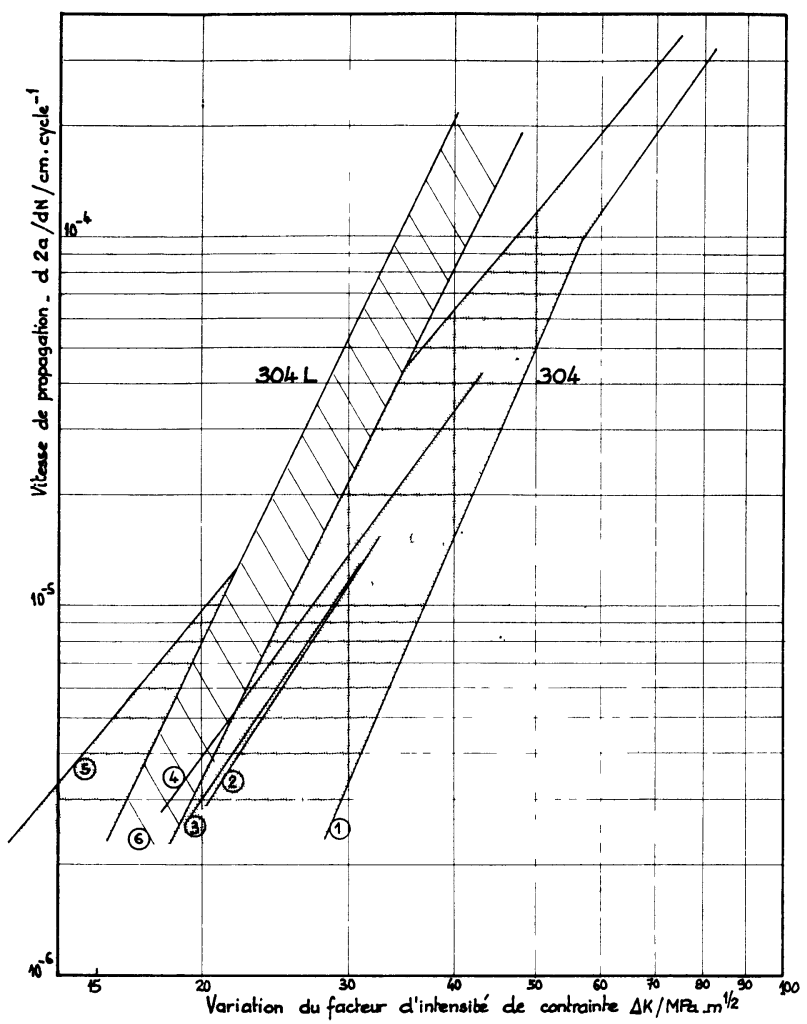

Fig. 7. - Comparaison des résultats $R=0$. Acier 304 et 304L. (1) Bathias, (2) Henry, (3) James, (4) Shahinian, (5) Brothers, (6) Cette étude.

Bathias [8]. Une comparaison des résultats pour $R=0$ est présentée sur la figure 7 . On note que les vitesses de propagation obtenues sur le 304L semblent plus élevées que celles indiquées pour le 304. A titre de comparaison le $\Delta K_{0}$ correspondant à $10^{-5} \mathrm{~cm}$ cycle $^{-1}$ vaut :

$$
\begin{aligned}
& \text { James Brothers Shahinian Henry Bathias Cette étude } \\
& \overline{28,6} \quad \overline{20,2} \quad \overline{27,05} \quad \overline{28,6} \quad \overline{36,8} \quad 21 \overline{21-25,1}
\end{aligned}
$$

3.2.3 Effet de la contrainte moyenne. - L'effet d'une contrainte moyenne ou de $R$ est assez marqué. De nombreuses formules ont été proposées pour tenir compte de $R$ faisant intervenir $R, K_{\mathrm{c}}, \Delta K$ seuil etc. [910]. Si l'on examine la variation du paramètre $C$ de la loi de Paris avec $R$ on note une dispersion très importante rendant difficile l'obtention d'une loi. En effet, une variation de $R$ entre différents essais peut être obtenue de diverses manières.

$$
R=\frac{\sigma_{\text {moy }}-\frac{\Delta \sigma}{2}}{\sigma_{\text {moy }}+\frac{\Delta \sigma}{2}} .
$$

La variation de $R$ peut être obtenue soit à $\sigma_{\text {moy }}$ constant en faisant varier $\Delta \sigma$, soit à $\Delta \sigma$ constant en faisant varier $\sigma_{\text {moy }}$.

Ceci correspond aux deux séries d'essais différentes effectuées sur le 304L et à une série globale effectuée sur le 316L. On constate que dans le second cas, l'influence de $R$ semble beaucoup plus réduite que dans le premier.

Si l'on fait varier $R$ à $\Delta \sigma$ constant, un $\Delta K$ donné est obtenu pour une longueur de fissure sensiblement constante et alors l'effet de $R$ est peu marqué. Par contre, si l'on fait varier $R$ par changement de $\Delta \sigma$, pour un $\Delta K$ donné, les longueurs de fissures sont différentes dans les différents essais et l'effet de $R$ est plus important.

On est donc amené à penser que l'effet de la longueur de fissure sur la vitesse de propagation est plus marqué que celui indiqué par la simple loi de Paris. On peut donc admettre que globalement l'exposant de la longueur de fissure doit être supérieur à $n / 2$ de manière à conduire à une loi du type

$$
\frac{\mathrm{d}^{2} a}{\mathrm{~d} N}=C(R) \Delta \sigma^{n} a^{m}
$$

ou

$$
C(R)(\Delta K)^{n} \cdot a^{P} \quad \text { avec } m>n / 2
$$

ce qui correspond globalement à une loi proposée par Mukkerjee Burns [11].

Les ajustements sont en cours pour préciser la validité de cette formulation.

4. Détermination du seuil de non-propagation. Le seuil de non-propagation a été déterminé pour les deux aciers. Une fissure a été considérée comme arrêtée

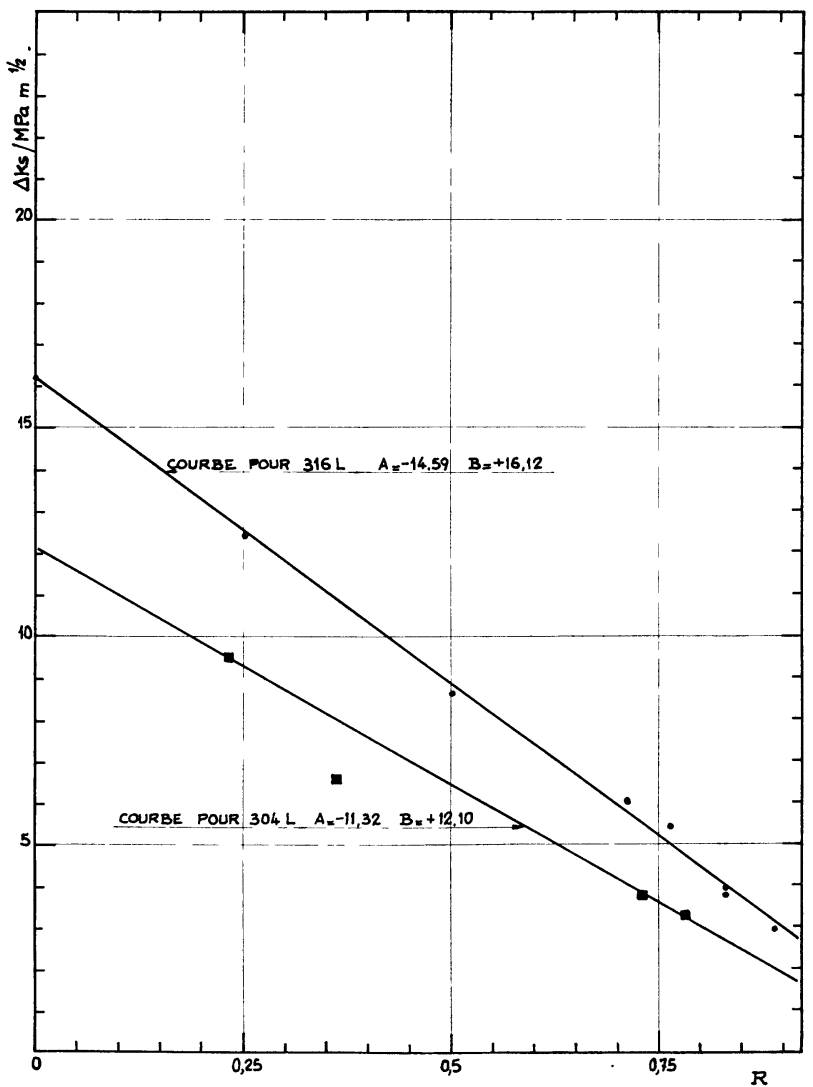

Fig. 8. - Variation de $\Delta K$ seuil avec $R: \Delta K_{\mathrm{s}}=A R+B$. 
si aucune propagation n'était mesurable après $5 \times 10^{6}$ cycles. Le seuil obtenu dépend du coefficient $R$ de manière décroissante illustrée sur la figure 8 .

Le seuil est nettement plus élevé pour l'acier 316L.

5. Conditions d'initiation. - L'entaille centrale utilisée présente un rayon de $0,1 \mathrm{~mm}$. Une fissure a été considérée comme initiée quand elle atteint en surface une longueur de $1 \mathrm{~mm}$. Le nombre de cycles nécessaires dépend du $\Delta K_{\mathrm{i}}$ initial $(\Delta K$ calculé comme si l'entaille était une fissure). Le résultat obtenu sur l'acier 304L est représenté sur la figure 9.

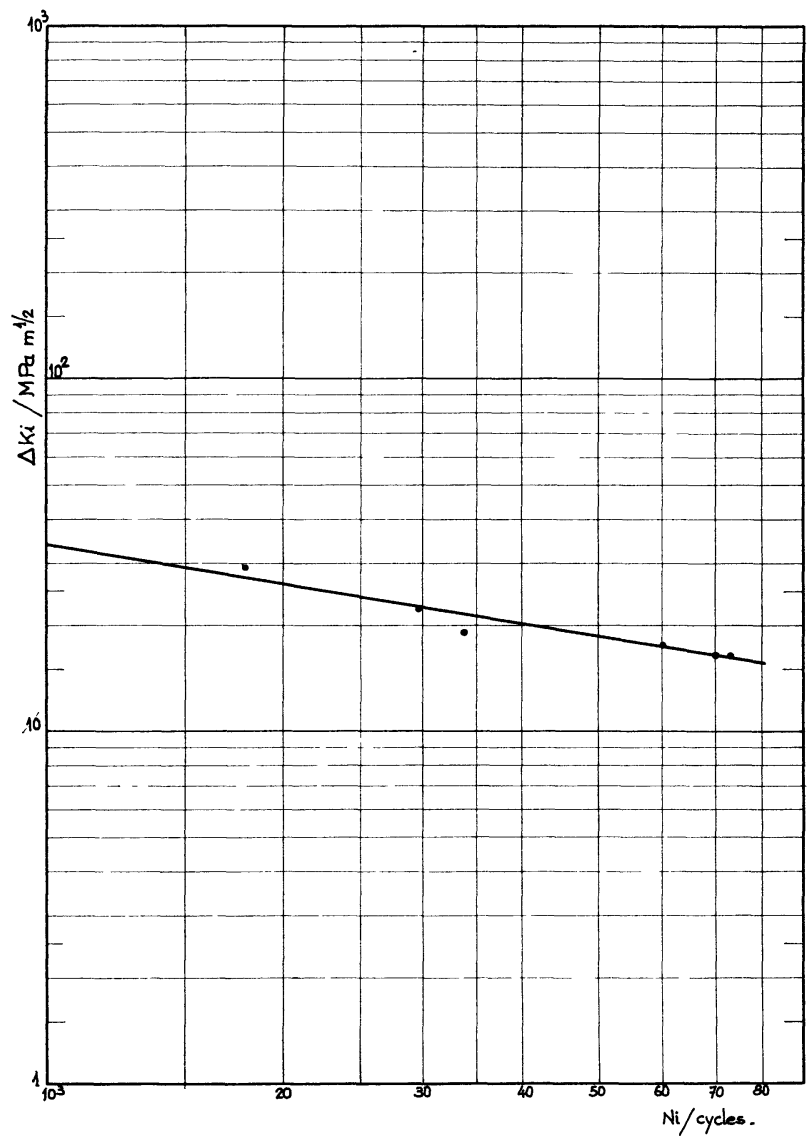

Fig. 9. $-\Delta K_{\mathrm{i}}$ en fonction du nombre de cycles à l'initiation (Fissure de $1 \mathrm{~mm}$ ). Rayon de 0,1 mm. Acier 304.

6. Examens des surfaces de rupture. - L'examen de la surface de rupture met nettement en évidence la zone fissurée par fatigue présentant des stries et la rupture finale à cupules obtenue par instabilité plastique. On a tenté de mesurer la vitesse de propagation microscopique par mesure d'interstries par examen au microscope à balayage et au microscope électronique.

Ces résultats partiels sont indiqués sur la figure 10 . On note un assez bon accord entre les mesures macroet microscopiques.

On remarque cependant sur les surfaces de rupture, des portions de fissuration intergranulaire illustrée sur la figure 11. En particulier, ce faciès intergranulaire a été observé au cours des essais d'arrêt de fissure

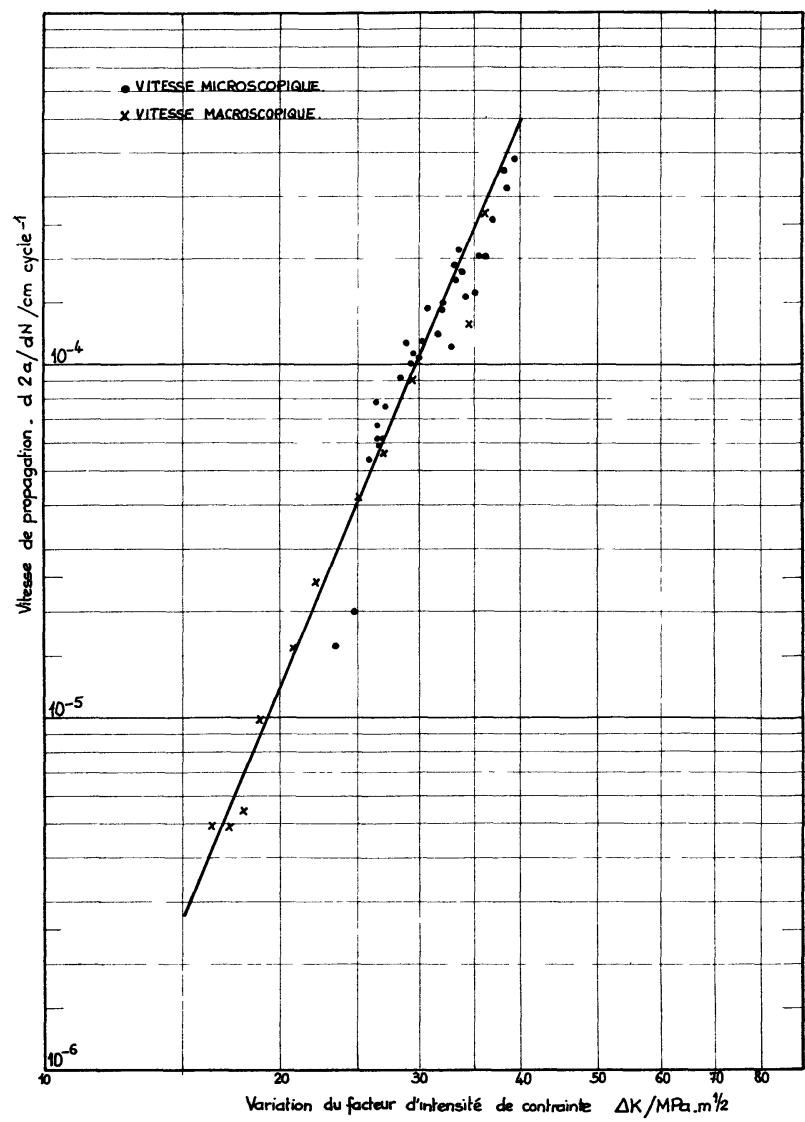

Fig. 10. - Comparaison des vitesses de fissuration macro- et microscopiques.

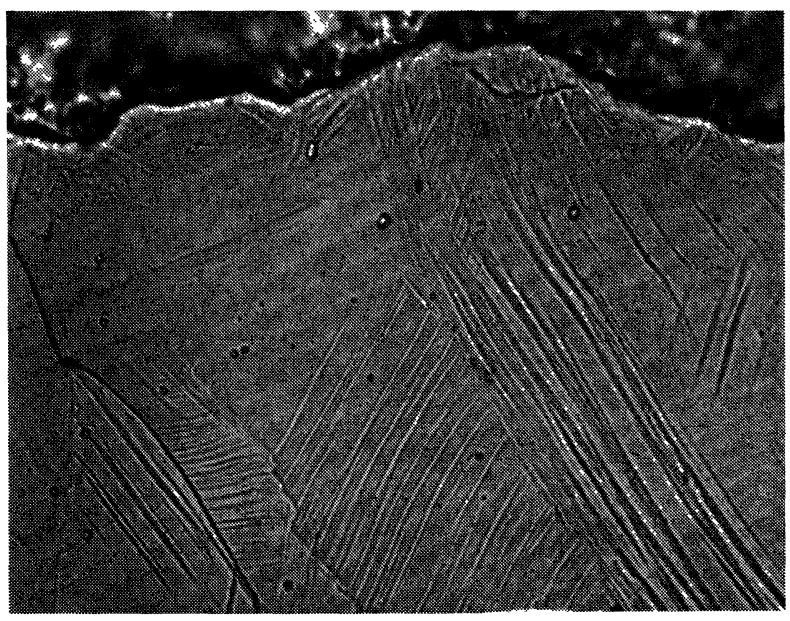

Fig. 11a. - Rupture intragranulaire et déformation plastique.

et semble correspondre aux vitesses de propagation très faibles.

7. Conclusion. Résumé. - Cette étude de la propagation de fissures par fatigue dans les aciers inoxydables austénitiques du type Z2CN1810 (304L) et Z2CND1712 (316L) a permis de dégager les points suivants. 


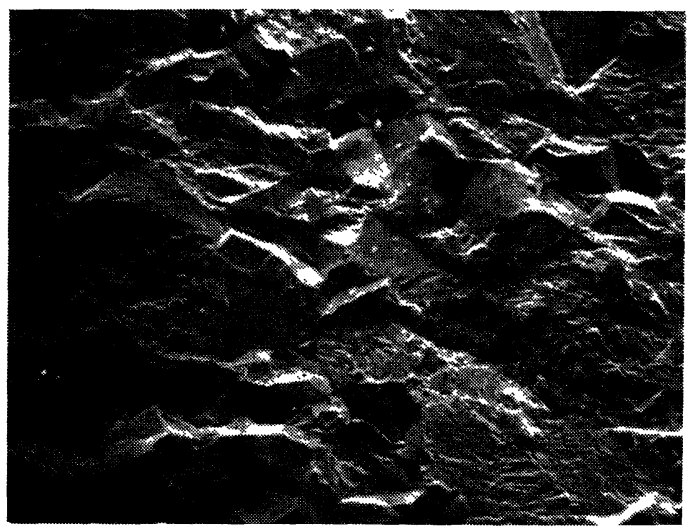

Fig. 11b. - Propagation de fissures dans le 304L.

7.1 Les vitesses de propagation des fissures par fatigue suivent globalement la loi de Paris

$$
\mathrm{d}^{2} a / \mathrm{d} N=C(\Delta K)^{n} .
$$

7.2 Dans les mêmes conditions de sollicitations, l'acier 304L présente une plus faible résistance à la propagation de fissure par fatigue que l'acier $316 \mathrm{~L}$.

7.3 L'influence de la contrainte moyenne est complexe. Il semble que la longueur de fissure inter- vienne d'une manière plus importante que ne le prédit la loi de Paris. Une formulation du type

$$
\frac{\mathrm{d}^{2} a}{\mathrm{~d} N}=C(R)(\Delta K)^{n} a^{P}
$$

semblerait mieux adaptée.

7.4 Par comparaison aux résultats présentés par divers auteurs concernant les aciers à carbone normal (304) on note une vitesse de propagation plus élevée dans les aciers à bas carbone étudiés ici (304L). La variation du facteur d'intensité de contrainte $\left(\Delta K_{0}\right)$ conduisant à une vitesse de propagation de $10^{-5} \mathrm{~cm}$ cycle $^{-1}$ est voisine de $23 \mathrm{MP} a \sqrt{\mathrm{m}}$ pour le $304 \mathrm{~L}$ et 27-28 pour le 304 .

7.5 On observe pour les 2 matériaux un seuil de nonpropagation (après $5 \times 10^{6}$ cycles) par fatigue $\left(\Delta K_{\mathrm{s}}\right)$. $\mathrm{Ce}$ seuil est généralement bas ( 5 à $10 \mathrm{MP} a \sqrt{\mathrm{m}})$. Il dépend d'une manière linéairement décroissante de $R$ et est plus élevé pour l'acier 316L que pour l'acier 304L.

7.6 La fissuration est généralement transcristalline. On rencontre cependant des zones de rupture intergranulaire qui correspondent à de très faibles vitesses de propagation c'est-à-dire proches des zones d'arrêt déterminant le $\Delta K_{\mathrm{s}}$.

\section{Bibliographie}

[1] Brown, W. F. et Srawley, J. E., Plane strain fracture toughness testing, ASTM STP 410 (1969) p. 11.

[2] FedDERSEN, cité dans Plane strain fracture toughness testing, ASTM STP 410 (1969) p. 79.

[3] Shahinian, P., Watson, H. E., et Smith, H. H., J. Materials 7 (1972) 527.

[4] Shahinian, P., Smith, H. H. et Watson, H. E., Fatigue at elevated temperature, ASTM STP 520 (1973).

[5] BRothers, A. J., Rapport : Fatigue crack growth in nuclear reactor piping steels, GEAP 5607 (1968).

[6] James, L. A. et Schwenk Jr, E. B., Metallurgical Transactions 2 (1971) 491.
[7] Henry, B., Bernard, J. et Gieb, Ph., 2nd International conference on structural mechanics in reactor technology, Berlin (1973), ref. F 4/7.

[8] Bathias, C. et Pelloux, R. M., Metallurgical Transactions, 4 (1973) 1265.

[9] Priddle, E. K., Rapport : Some equations describing constant amplitude fatigue crack propagation characteristics of a mild steel, CEGB/RD/B/N 2390 (1972).

[10] McEvily, A. J., Kumble, R. et Donahue, R. J., 3rd International Congress on fracture, Munich (1973), Verein Deutscher Eisen huttenleute, Dusseldorf.

[11] MukherJeE, B. et Burns, D. J., ASTM STP 511. 